ORIGINAL ARTICLE

\section{Mucus Clearance and Lung Function in Cystic Fibrosis with Hypertonic Saline}

\author{
Scott H. Donaldson, M.D., William D. Bennett, Ph.D., Kirby L. Zeman, Ph.D.,
} Michael R. Knowles, M.D., Robert Tarran, Ph.D., and Richard C. Boucher, M.D.

\author{
ABSTRACT
}

\section{BACKGROUND}

Abnormal homeostasis of the volume of airway surface liquid in patients with cystic fibrosis is thought to produce defects in mucus clearance and airway defense. Through osmotic forces, hypertonic saline may increase the volume of airway surface liquid, restore mucus clearance, and improve lung function.

\section{METHODS}

A total of 24 patients with cystic fibrosis were randomly assigned to receive treatment with inhaled hypertonic saline $(5 \mathrm{ml}$ of 7 percent sodium chloride) four times daily with or without pretreatment with amiloride. Mucus clearance and lung function were measured during 14-day baseline and treatment periods.

\section{RESULTS}

Long-term inhalation of hypertonic saline without pretreatment with amiloride (i.e., with placebo pretreatment) resulted in a sustained ( $\geq 8$ hours) increase in 1-hour rates of mucus clearance, as compared with those with amiloride pretreatment (14.0 \pm 2.0 vs. $7.0 \pm 1.5$ percent, respectively; $\mathrm{P}=0.02$ ) and increased 24 -hour rates of mucus clearance over baseline. Furthermore, inhalation of hypertonic saline with placebo improved the forced expiratory volume in one second $\left(\mathrm{FEV}_{1}\right)$ between the baseline period and the treatment period (mean difference, 6.62 percent; 95 percent confidence interval, 1.6 to $11.7 ; \mathrm{P}=0.02$ ), whereas hypertonic saline with amiloride did not improve $\mathrm{FEV}_{1}$ (mean difference, 2.9 percent; 95 percent confidence interval, -2.2 to $8.0 ; \mathrm{P}=0.23$ ). Forced vital capacity (FVC), the forced expiratory flow between 25 and 75 percent of FVC $\left(\mathrm{FEF}_{25-75}\right)$, and respiratory symptoms also significantly improved in patients treated with hypertonic saline and placebo, whereas the residual volume as a proportion of total lung capacity (RV:TLC) did not change in either group. A comparison of the changes in lung function in the two groups showed no significant difference. In vitro data suggested that sustained hydration of airway surfaces was responsible for the sustained improvement in mucus clearance, whereas inhibition of osmotically driven water transport by amiloride accounted for the observed loss of clinical benefit.

\section{CONCLUSIONS}

In patients with cystic fibrosis, inhalation of hypertonic saline produced a sustained acceleration of mucus clearance and improved lung function. This treatment may protect the lung from insults that reduce mucus clearance and produce lung disease.
From the University of North Carolina at Chapel Hill Cystic Fibrosis Research and Treatment Center (S.H.D., W.D.B., M.R.K., R.T., R.C.B.) and the Center for Environmental Medicine, Asthma and Lung Biology (W.D.B., K.L.Z.) — both in Chapel Hill. Address reprint requests to Dr. Donaldson at 6019 Thurston Bowles Bldg., CB\# 7248, University of North Carolina at Chapel Hill Research and Treatment Center, Chapel Hill, NC 27599, or at scott_donaldson@med. unc.edu.

Drs. Donaldson and Bennett contributed equally to this article.

N Engl J Med 2006;354:241-50. Copyright (c) 2006 Massachusetts Medical Society. 
M

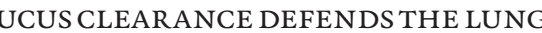
against inhaled bacteria. The efficiency of mucus clearance depends on an adequate volume of airway surface liquid (i.e., hydration). ${ }^{1}$ One hypothesis for the pathogenesis of lung disease in patients with cystic fibrosis is that a lack of regulation of sodium absorption and chloride secretion causes depletion of airway surface liquid, slows mucus clearance, and promotes the formation of adherent mucus plaques on airway surfaces. Mucus plaques and plugs obstruct airways and provide the nidus for infection..$^{2,3}$

On the basis of this hypothesis, therapies that increase the volume of airway surface liquid, and hence mucus clearance, should improve lung disease in patients with cystic fibrosis. Inhaled hypertonic saline has been shown to produce shortterm stimulation of mucus clearance $e^{4,5}$ and, in separate studies, to improve lung function..$^{6,7}$ In vitro studies with normal human airway epithelia demonstrated that hypertonic saline increases the volume of airway surface liquid, but the effects were transient and, therefore, predicted to be of limited therapeutic benefit. ${ }^{8}$ These in vitro studies, however, demonstrated that slowing the absorption of sodium with amiloride, a sodiumchannel blocker, significantly extended the duration of the increase in the volume of airway surface liquid. We tested the hypothesis that pretreatment with amiloride would extend the duration of hypertonic saline-induced acceleration of mucus clearance and enhance improvement in lung function in patients with cystic fibrosis.

\section{METHODS}

\section{STUDY DESIGN}

The study protocol was approved by the University of North Carolina Committee on the Protection of Rights of Human Subjects, and written informed consent was obtained. Patients were enrolled between January 2001 and February 2004. Inclusion criteria included an established diagnosis of cystic fibrosis, an age of at least 14 years, and a forced expiratory volume in one second $\left(\mathrm{FEV}_{1}\right)$ of 50 percent or more of the predicted value after bronchodilation. Exclusion criteria included unstable lung disease (as evidenced by the administration of intravenous antibiotics within four weeks before screening, a change of medical regimen within two weeks before screening, or an $\mathrm{FEV}_{1} \geq 15$ percent below the best clinical value during the previous six months), evidence of reactive airways ( $>15$ percent response to bronchodilation or clinical diagnosis of asthma), and the use of hypertonic saline within two weeks before screening. (For details, see the Supplementary Appendix, which is available with the full text of this article at www.nejm.org.)

After screening, patients with cystic fibrosis entered a 14-day baseline observation period (Fig. 1), after which they were randomly assigned in a 1:1 distribution to pretreatment with either amiloride (at a dose of $1 \mathrm{mg}$ per milliliter in $4.5 \mathrm{ml}$ of 0.12 percent sodium chloride [Sifavitor]) or taste-masked placebo (at a dose of $0.25 \mathrm{mg}$ of quinine sulfate per milliliter in $4.5 \mathrm{ml}$ of 0.12 percent sodium chloride [DSM Minera]) before the administration of 7 percent sodium chloride (at a dose of $5 \mathrm{ml}$ ). Aerosols were delivered four times daily with the use of a Pari LC Star nebulizer and Pari Proneb Ultra compressor (Pari). Albuterol (at a dose of $180 \mu \mathrm{g}$ ) was administered with a metered-dose inhaler with a spacer 30 to 60 minutes before the administration of study medications.

\section{STUDY OUTCOMES}

The prespecified outcomes of the primary study were the percentage changes in $\mathrm{FEV}_{1}$ and forced vital capacity (FVC) during the treatment period. Secondary outcomes were the rates of mucus clearance, the forced expiratory flow between 25 and 75 percent of FVC $\left(\mathrm{FEF}_{25-75}\right.$ ), the ratio of residual volume to total lung capacity (RV:TLC), and quality of life. The latter was measured with the Cystic Fibrosis Questionnaire for teens and adults, in which domain scores range from 0 to 100 , with higher scores reflecting better perceived health status. ${ }^{9}$

Whole-lung mucus clearance was measured, as previously described, ${ }^{10,11}$ four times during the study: during the baseline interval (basal mucus clearance on day 13), immediately after the first dose of study medications (mucus clearance after the first dose on day 15), during long-term use of study medication but eight or more hours after administration of the preceding dose to assess sustained effects (durability of mucus clearance on day 26), and immediately after the administration of study medications at the end of the treatment interval (mucus clearance after the last dose on day 28). The 1-hour rate of mucus clear- 
ance, calculated from the average of measurements at 10-minute intervals, served as the primary index of mucociliary clearance. Patients returned 24 hours after the administration of radioisotopes to measure the cumulative mucus clearance.

For comparison, data describing mucus clearance were collected from 15 healthy, nonsmoking adults (mean $[ \pm S D]$ age, $25.6 \pm 3.9$ years; $\mathrm{FEV}_{1}$, $103 \pm 9.8$ percent of the predicted value) with the use of identical techniques, except that healthy subjects received pretreatment with normal saline $(2.5 \mathrm{ml}$ over a period of 30 minutes, delivered by de Vilbiss 646 nebulizer), rather than with albuterol. Neither the administration of albuterol in patients with cystic fibrosis nor saline by nebulizer in controls was expected to affect mucus clearance. ${ }^{12}$

Spirometric and plethysmographic measurements of lung volumes were performed according to American Thoracic Society standards, ${ }^{13}$ and the percentage changes during baseline and treatment periods were calculated. Spirometric measurement was also performed two hours after the administration of study medications on day 15 and day 28 and compared with values before administration. Quantitative sputum cultures and serum chemical profiles were performed before and at completion of the treatment period.

In vitro experiments were performed using bronchial epithelia obtained and cultured as previously described. ${ }^{2}$ Airway surface liquid was labeled with $2 \mathrm{mg}$ of Texas red dextran per milliliter in $20 \mu \mathrm{l}$ of phosphate-buffered saline, and the volume of airway surface liquid was monitored by axial confocal microscopy. ${ }^{2}$ Sodium chloride (at a dose of $0.8 \mathrm{mg}$ ) with or without amiloride (final concentration, $400 \mu \mathrm{M}$ ) was added (in perfluorocarbon) to the mucosal surface of epithelial cultures from patients with cystic fibrosis and from controls. The volume of airway surface liquid was serially measured before and after the addition of sodium chloride. The contribution of chloride transport by the cystic fibrosis transmembrane conductance regulator (CFTR) to the change in the volume of airway surface liquid in normal epithelia after the administration of sodium chloride was assessed by pretreating cultures with a CFTR inhibitor (CFTR $_{\text {inh }}-172$ at a concentration of $5 \mu \mathrm{M})^{14}$ or by substituting the impermeant anion gluconate for chloride (3.0 mg of sodium gluconate with $20 \mathrm{mM}$ of calcium chloride to maintain calcium activity ${ }^{15}$ ).

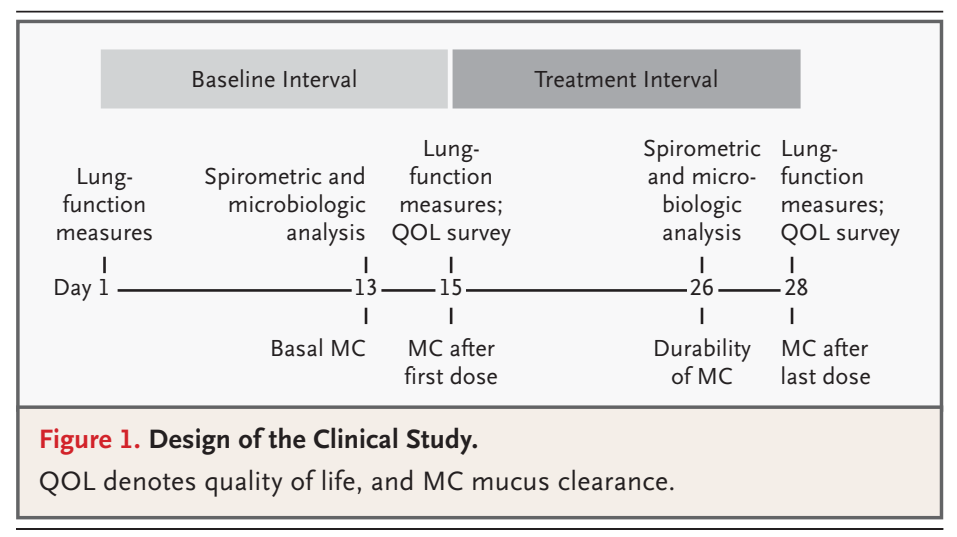

The effect of amiloride on transepithelial water transport was measured by the addition of mannitol (at a concentration of $300 \mathrm{mM}$ in $100 \mu \mathrm{l}$ of phosphate-buffered saline) with or without amiloride (at a concentration of $400 \mu \mathrm{M}$ ) to the apical surface of airway epithelial cultures from patients with cystic fibrosis to generate an osmotic driving force. Water flow was quantitated by measuring the resulting change in serosal bath fluorescence. ${ }^{16}$ (Additional in vitro methods are described in the Supplementary Appendix.)

\section{STATISTICAL ANALYSIS}

Sample size was based on the ability to detect a treatment effect equal to $1 \mathrm{SD}$ of the $\mathrm{FEV}_{1}$. A sample size of 16 patients per group provided 80 percent power to detect this treatment effect with the use of a two-sided test $(\alpha=0.05)$. We anticipated a reduced variance and an increase in power because two $\mathrm{FEV}_{1}$ measures were averaged to produce each end of interval values.

Univariate data analyses were performed with the use of paired or unpaired two-sided t-tests, as appropriate, with a P value of 0.05 or less accepted as indicating significance. Patients who had undergone randomization and who had received at least one dose of study medication and contributed any data during the treatment interval were included in a modified intention-to-treat analysis.

For studies of mucus clearance, the ratio of particle deposition in central regions of the lung, as compared with peripheral regions (C:P ratio), was calculated for each mucus-clearance scan. The 1-hour and 24-hour mucus-clearance rates were calculated and compared with use of paired and unpaired t-tests, as appropriate. The effect of study treatments on mucus clearance was also 
tested with a mixed-model analysis of three components of variance that included the study visit, group assignment, and the C:P ratio as explanatory variables. Differences that are reported as significant were supported by both statistical methods.

\section{RESULTS}

DEMOGRAPHIC CHARACTERISTICS

Of the 29 patients who underwent screening, 27 met eligibility criteria and were enrolled. Three subjects were withdrawn before randomization because of pulmonary exacerbations (two patients) or hyperkalemia (one patient). At baseline, the treatment groups were well matched (Table 1). Three patients who underwent randomization did not contribute data to treatment-interval end points owing to adverse events and were not included in the modified intention-to-treat analysis. These events included a pulmonary exacerbation (in the group receiving hypertonic saline with placebo); probable abdominal sepsis in a patient with immunosuppression after liver transplantation (in the group receiving hypertonic saline with amiloride); and an 18.6 percent drop in the $\mathrm{FEV}_{1}$ two hours after the administration of the first dose of study drugs (in the group receiving hypertonic saline with amiloride), which was a predefined criterion for exclusion. Other adverse events were rare or mild in severity (as listed in the Supplementary Appendix).

\section{BASAL MUCUS CLEARANCE}

The mean $( \pm S E)$ one-hour mucus-clearance rate, which is dominated by large-airway clearance, did not differ significantly between patients with cystic fibrosis $(9.3 \pm 1.1$ percent) and controls (10.0 \pm 1.7 percent) (mean difference, 0.6 ; 95 percent confidence interval, -3.3 to $4.7 ; \mathrm{P}=0.73$ ) (Fig. $2 \mathrm{~A}$ and $2 \mathrm{~B}$ ). However, mucus clearance at 24 hours was reduced in patients with cystic fibrosis, as compared with controls $(40.7 \pm 2.5$ vs. $53.5 \pm 2.8$ percent, $\mathrm{P}<0.001)$. This difference in the 24-hour measure did not reflect altered tracer deposition, as the C:P mean $( \pm \mathrm{SE})$ ratios of particle deposition did not differ significantly between patients with cystic fibrosis $(1.48 \pm 0.05)$ and controls $(1.57 \pm$ 0.06) (mean difference, 0.09 ; 95 percent confidence interval, -0.07 to $0.25 ; \mathrm{P}=0.26$ ).

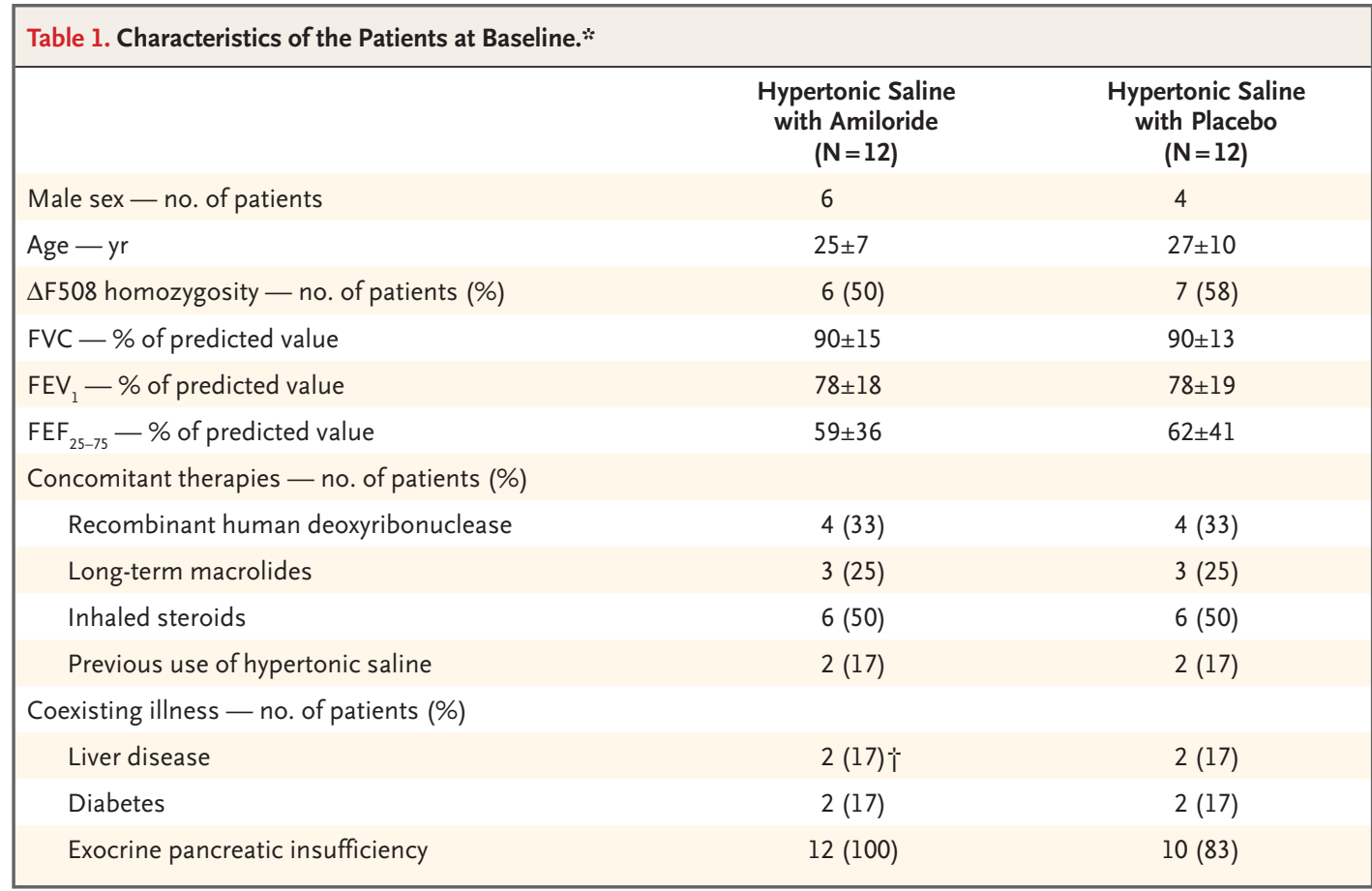

* Plus-minus values are means \pm SD. FVC denotes forced vital capacity, $\mathrm{FEV}_{1}$ forced expiratory volume in one second, and $\mathrm{FEF}_{25-75}$ forced expiratory flow between 25 and 75 percent of FVC.

$\uparrow$ Patients underwent liver transplantation. 


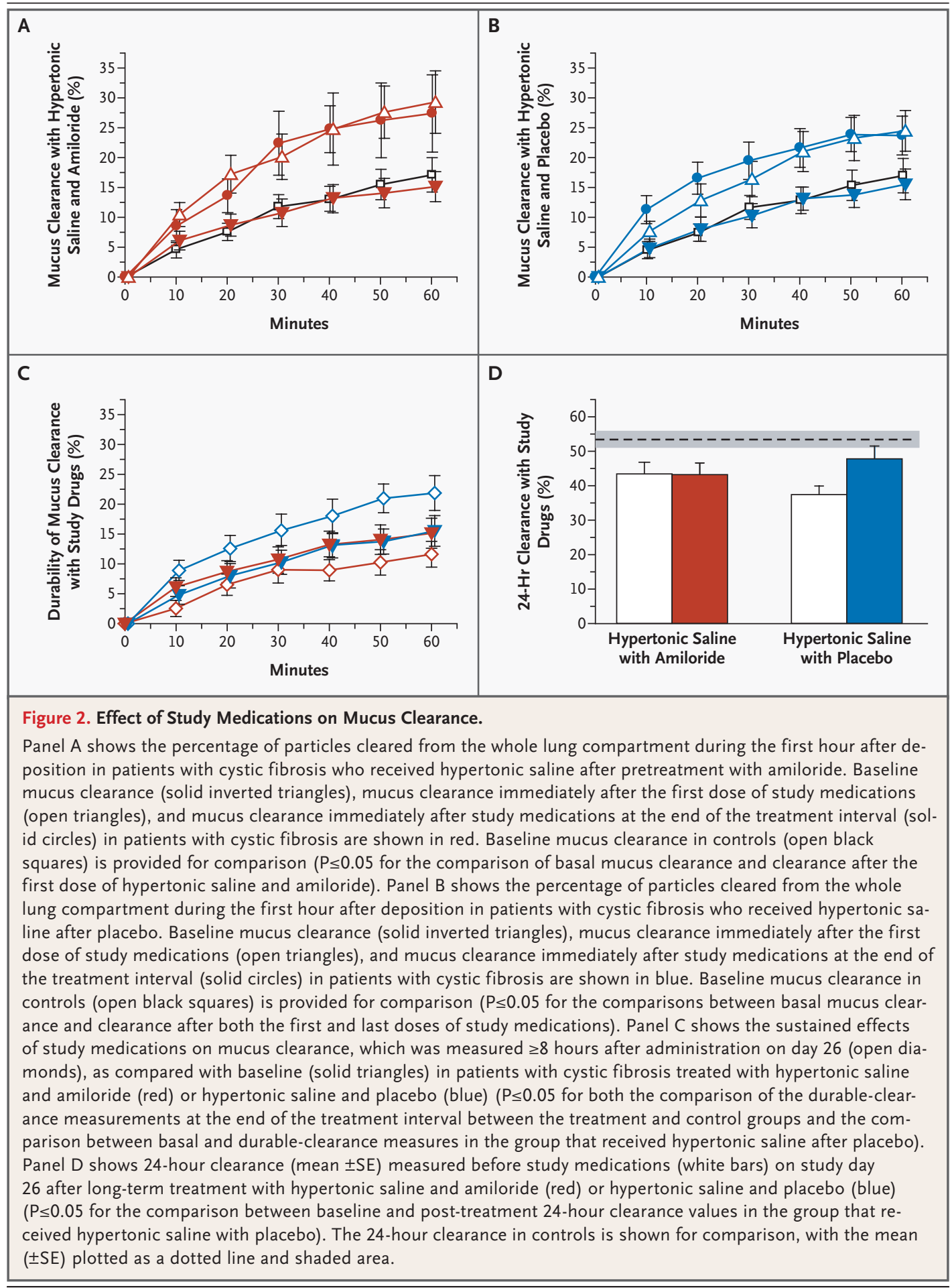

EFFECT OF HYPERTONIC SALINE WITH OR WITHOUT AMILORIDE

Baseline 1-hour mucus-clearance rates and cumulative mucus clearance at 24 hours (on day 13) did not differ in the two treatment groups (Fig. 2).
The one-hour mucus-clearance rate that was measured immediately after the first dose of study medications (on day 15) was significantly increased relative to baseline in both groups $(\mathrm{P}=0.01$ and $\mathrm{P}=0.05$ in groups receiving hypertonic saline with 
amiloride and with placebo, respectively) (Fig. $2 \mathrm{~A}$ and $2 \mathrm{~B}$ ). The one-hour mucus-clearance rate that was measured immediately after study medications on the final day of treatment (on day 28) was similarly increased relative to basal mucus clearance, suggesting no diminution in the peak effects on mucus clearance over this two-week period (Fig. 2A and 2B).

There were two important differences in the effects of treatment regimen on mucus clearance during long-term administration. First, the assessment of the durability of mucus clearance revealed a significantly faster one-hour mucusclearance rate in the group receiving hypertonic saline with placebo than in the group receiving hypertonic saline with amiloride $(14.0 \pm 2.0$ vs. $7.0 \pm 1.5$ percent, $\mathrm{P}=0.02$ ) (Fig. $2 \mathrm{C}$ ). This difference reflected the fact that only hypertonic saline with placebo produced a sustained increase ( $\geq 8$ hours) in the durability measurement of onehour mucus clearance (14.0 \pm 2.0 percent) versus that for the basal measurement of mucus clearance (9.3 \pm 1.6 percent; $\mathrm{P}=0.04$ ) (Fig. $2 \mathrm{C}$ ). Second, although the cumulative 24-hour mucus-clearance rate was not significantly higher in the group that received hypertonic saline with placebo at the durability-of-mucus-clearance scan (mean difference, 4.6 percent; 95 percent confidence interval, -5.9 to $15.1 ; \mathrm{P}=0.36$ ), long-term administration of hypertonic saline with placebo increased the 24-hour rate as compared with pretreatment values $(\mathrm{P}=0.04)$, whereas long-term administration of hypertonic saline with amiloride did not increase the rate $(\mathrm{P}=0.49)$ (Fig. 2D).

\section{LUNG FUNCTION}

Similar small changes in lung function were observed during the baseline interval in both treatment groups (Fig. 3). The absolute percentage changes in lung function during the treatment interval favored the group that received hypertonic saline with placebo but were not significantly different (mean $\mathrm{FEV}_{1}$ difference, 4.7 percent; 95 percent confidence interval, -1.3 to 10.6; $\mathrm{P}=0.12$; mean FVC difference, 1.8 percent; 95 percent confidence interval, -1.9 to $5.5 ; \mathrm{P}=0.23$; mean $\mathrm{FEF}_{25-75}$ difference, 13.1 percent; 95 percent confidence interval, -1.4 to $27.7 ; \mathrm{P}=0.07$ ). The corresponding absolute increases in $\mathrm{FEV}_{1}$ resulting from treatment were $147 \pm 39 \mathrm{ml}$ for the group receiving hypertonic saline with placebo and $34 \pm 66 \mathrm{ml}$ for the group receiving hypertonic

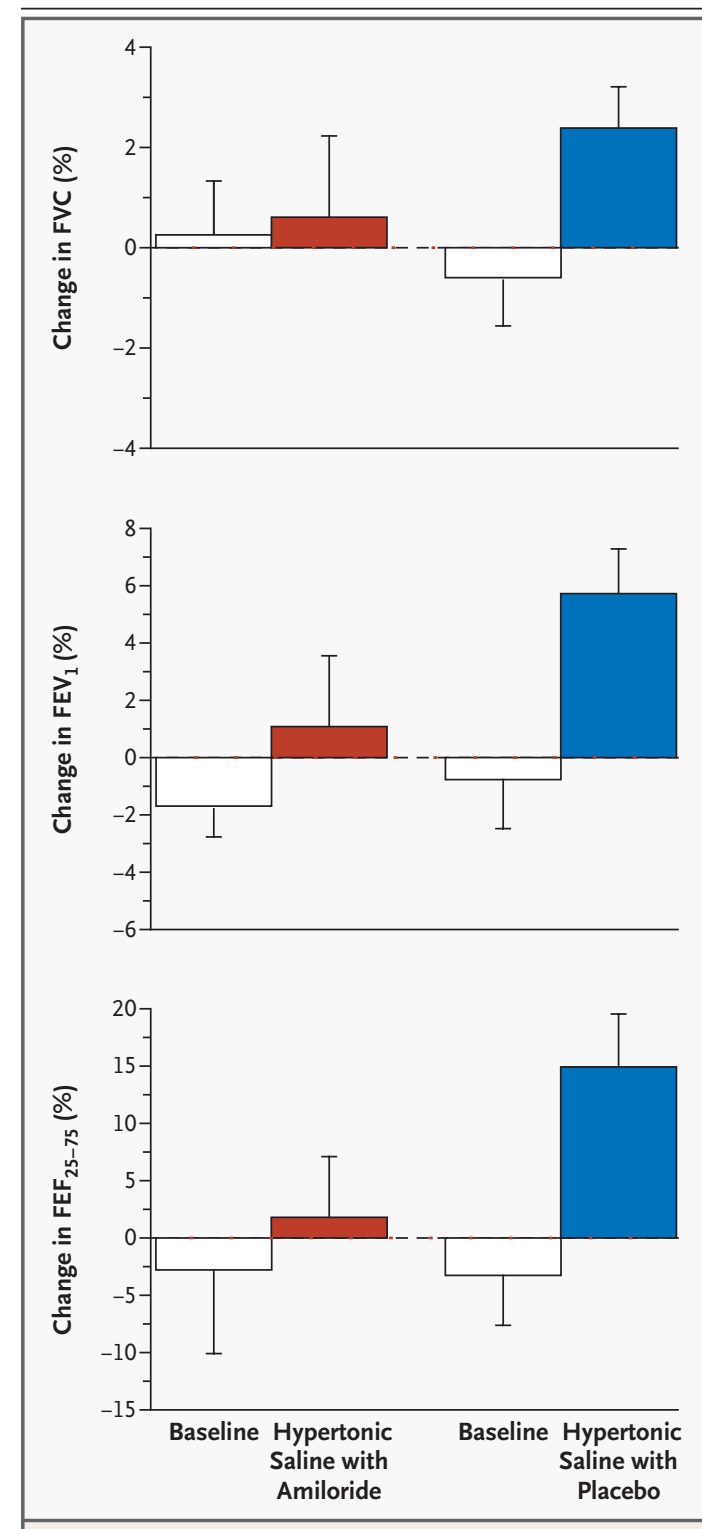

Figure 3. Effect of Study Medications on Lung Function. The mean $( \pm \mathrm{SE})$ percentage change in FVC, the $\mathrm{FEV}_{1}$, and the $\mathrm{FEF}_{25-75}$ during the baseline (white bars) and treatment intervals are shown for each treatment group, indicating the results of administration of hypertonic saline with amiloride (red) and with placebo (blue) $(\mathrm{P}<0.05$ for comparisons between baseline and posttreatment measures in the group receiving hypertonic saline after placebo). T bars indicate standard errors.

saline with amiloride (mean difference, $113 \mathrm{ml}$; 95 percent confidence interval, -44 to 271). However, comparisons of changes in lung function during the treatment and baseline intervals revealed significant improvements in FVC ( $\mathrm{P}=0.05)$, $\mathrm{FEV}_{1}(\mathrm{P}=0.02)$, and $\mathrm{FEF}_{25-75}(\mathrm{P}=0.02)$ in the group 
that received hypertonic saline with placebo but no differences in the group that received hypertonic saline with amiloride $(\mathrm{P}=0.83, \mathrm{P}=0.23$, and $\mathrm{P}=0.55$, respectively) (Fig. 3). No significant differences were observed in RV:TLC ratios.

\section{QUALITY OF LIFE}

The mean $( \pm \mathrm{SE})$ respiratory symptom score as measured after the treatment interval was significantly better in the group that received hypertonic saline with placebo than in the group that received hypertonic saline with amiloride $(82.3 \pm 3.1$ vs. $70.0 \pm 3.1, \mathrm{P}=0.01)$. Significant improvement in respiratory symptoms but a higher perceived workload or burden related to medical treatment was also observed in a comparison of scores at baseline and during treatment in the group that received placebo but not in the group that received amiloride (complete questionnaire results are available in the Supplementary Appendix).

\section{SAFETY AND ADVERSE EVENTS}

No treatment-related serious adverse events occurred. Mean ( $\pm \mathrm{SE})$ absolute changes in $\mathrm{FEV}_{1}$ two hours after the administration of study medication on day $15(0.85 \pm 3.79$ percent in the placebo group and $-2.47 \pm 6.66$ percent in the amiloride group) and on day $28(-0.55 \pm 4.46$ percent in the placebo group and $-1.39 \pm 3.85$ percent in the amiloride group) were small and not significantly different between groups. Serum chemical analyses, hematologic values, and quantitative sputum cultures (for total bacteria and pseudomonas) did not change with either treatment (see the Supplementary Appendix).

\section{IN VITRO RESPONSE TO HYPERTONIC SALINE}

The addition of sodium chloride to the mucosal surface of airway epithelial cultures, simulating deposition of 7 percent sodium chloride, ${ }^{10}$ produced a much larger and more sustained increase in the volume of airway surface liquid in airway cultures from patients with cystic fibrosis than in cultures from controls (Fig. 4A and 4B). We tested the hypothesis that the sustained response in cultures from patients with cystic fibrosis reflected the absence of the CFTR chloride channel as a route for transcellular salt absorption. Control cultures had a significantly increased response in the volume of airway surface liquid to hypertonic challenge when pretreated with a CFTR chloride-channel blocker (CFTR $\left._{\text {inh }}-172\right)$ or with replacement of chloride in the apical hypertonic solution with an impermeant anion (Fig. 4B). These data indicate that chloride transport through CFTR is a key determinant of the response in the volume of airway surface liquid to the administration of hypertonic saline.

\section{INHIBITION OF TRANSEPITHELIAL WATER}

TRANSPORT BY AMILORIDE

The capacity of hypertonic saline to produce large and prolonged increases in the volume of airway surface liquid in airway epithelial cultures of patients with cystic fibrosis was markedly reduced by pretreatment with amiloride (Fig. 4A and 4C). The slower increase in serosal fluorescence in response to an apical hyperosmolar mannitol solution suggested that the reduced response in the volume of airway surface liquid to hypertonic saline after amiloride pretreatment reflected a block of transepithelial water flow (Fig. 4D). This effect was not specific to epithelia from patients with cystic fibrosis, since amiloride also slowed osmotically driven water flow in control epithelia; the rate of increase in fluorescence was reduced by $74.6 \pm 4.0$ percent in controls, as compared with $89.8 \pm 2.3$ percent in patients with cystic fibrosis $(\mathrm{P}=0.01)$.

The action of amiloride to block the water permeability of airways was further characterized. First, the 50 percent inhibitory concentration $\left(\mathrm{IC}_{50}\right)$ for amiloride inhibition of water permeability was $6 \mu \mathrm{M}$, a value that is less potent by a factor of 10 than that for inhibition of the epithelial sodium channel. Second, a cellular site of action was indicated by experiments in which a blocker of cellular aquaporins, mercuric chloride, mimicked the blockade by amiloride of hypertonic saline-induced expansion of airway surface liquid; amiloride also inhibited hypertonic saline-induced cell shrinkage. Finally, the amiloride blockade was pharmacologically specific, since an analogue that more potently inhibits the epithelial sodium channel (benzamil) was active, whereas an analogue more specific for the sodium-hydrogen exchanger (dimethylamiloride) was not (additional in vitro data are available in the Supplementary Appendix).

\section{DISCUSSION}

Treatment of patients who have cystic fibrosis with inhaled hypertonic saline after placebo re- 


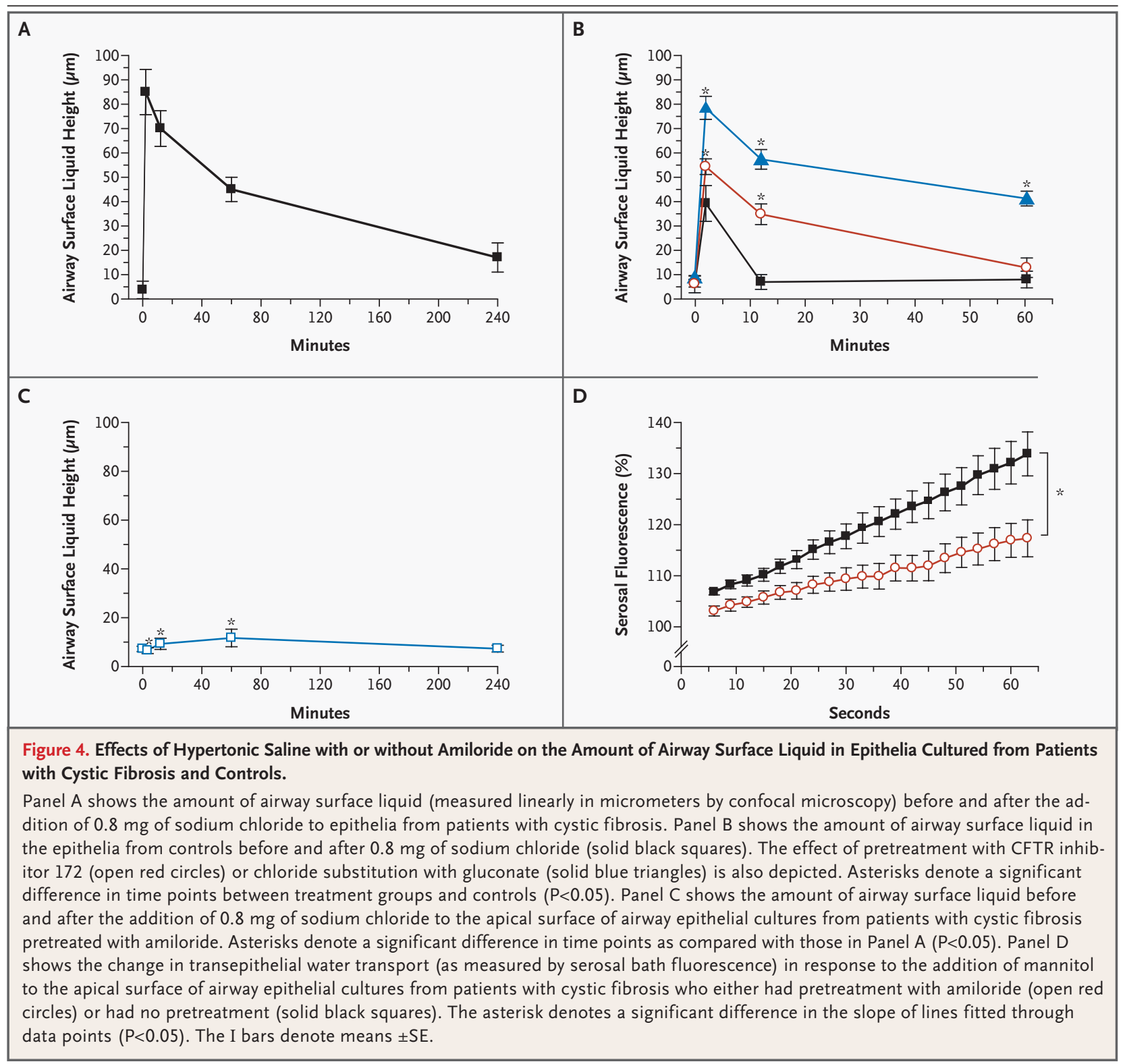

sulted in a sustained increase in the one-hour mucus-clearance rate and was associated with improvements in lung function and respiratory symptoms over baseline values. In contrast, patients who were treated with hypertonic saline after amiloride had no sustained increase in mucus clearance and no improvement in lung function or respiratory symptoms. The relative effectiveness of hypertonic saline treatment alone, as compared with the administration of hypertonic saline after pretreatment with amiloride, was contrary to our hypothesis. These findings raised two questions: Why was the effect of hypertonic saline on mucus clearance so prolonged in patients with cystic fibrosis as compared with controls? ${ }^{8}$ And why did amiloride blunt the ability of hypertonic saline to produce sustained increases in mucus clearance? We investigated these questions in airway cultures because a tight linkage between the volume of airway surface liquid and mucus transport has been demonstrated in this model system. ${ }^{1}$

The administration of hypertonic saline in vitro produced a larger and more sustained increase in the volume of airway surface liquid in cultures from patients with cystic fibrosis than in 
cultures from controls (Fig. 4A and 4B). The role of CFTR in the dissipation of the high luminal salt concentration that is generated by hypertonic saline deposition was revealed with pharmacologic blockade of CFTR and chloride-replacement studies (Fig. 4B). The resulting prolongation of an osmotic driving force would draw more water onto the airway surface in patients with cystic fibrosis, increase the volume of airway surface liquid, and produce a sustained increase in mucus clearance.

The effect of amiloride in blunting clinical improvements with hypertonic saline might also be explained by effects on the volume of airway surface liquid. In vitro experiments revealed markedly reduced expansion of airway surface liquid in response to the administration of hypertonic saline after pretreatment with amiloride (Fig. 4C) owing to the blockade of apical membrane water permeability (Fig. 4D and the Supplementary Appendix). As a result, the administration of hypertonic saline with amiloride may increase the tonicity of airway surface liquid, which through electrostatic effects on mucins may account for the acute increase in mucus clearance. ${ }^{17,18}$ It also may limit the expansion in the volume of airway surface liquid, preventing the sustained increase in mucus clearance and improvement in lung function observed with hypertonic saline alone.

In another article in this issue of the Journal, Elkins et al. ${ }^{19}$ also observed a modest improvement in lung function with inhaled hypertonic saline but observed a striking reduction in pulmonary exacerbations. We propose that hypertonic saline produced the sustained airway surface hydration required to clear retained mucus, but its efficacy was limited by its failure to reach many obstructed airways, as evidenced by the moderate resulting increase in $\mathrm{FEV}_{1} \cdot{ }^{19}$ In contrast, effective delivery of hypertonic saline to relatively nonob- structed airways produced supranormal 1-hour mucus-clearance rates and improved cumulative 24-hour mucus-clearance rates. We speculate that the durable increase in mucus clearance protected relatively nonobstructed lung regions from exogenous insults (e.g., viral infections ${ }^{20,21}$ ) that slow mucus clearance and hence promote intrapulmonary spread of bacterial infection or the development of new mucus obstruction, thus accounting for the large reduction in the exacerbation rate observed by Elkins et al.

In summary, inhalation of hypertonic saline four times daily provides a modest improvement in lung function and respiratory symptoms without substantial adverse events. On the basis of studies of airway epithelia in vitro, it appears likely that the mechanism of action of hypertonic saline is to provide long-term hydration of airway surfaces and promote a sustained increase in mucus clearance. This study points to a vital therapeutic role for the restoration of hydration to airway surfaces in patients with cystic fibrosis and suggests that measures of sustained mucus clearance may serve as a useful surrogate outcome for future drug development.

Supported by a grant (DONALD00A0, to Dr. Donaldson) from the Cystic Fibrosis Foundation, by National Institutes of Health (NIH) grants (1K08 HL68617, P50-HL060280, and 5-P01-HL34322), and by an NIH grant (RR00046) from the General Clinical Research Center program of the Division of Research Resources.

Drs. Knowles and Boucher report having received consulting fees from Inspire Pharmaceuticals and having equity interests in Inspire Pharmaceuticals and Parion Sciences. Dr. Boucher reports having an equity interest in Respirics. Dr. Boucher was named coinventor on a patent for dry-powder amiloride, which was subsequently licensed to Parion Sciences. He also holds multiple other patents on compounds designed to treat lung diseases. No other potential conflict of interest relevant to this article was reported.

We are indebted to Drs. Paul Stewart and Lisa LaVange of the Biostatistics Department at the University of North Carolina School of Public Health, who provided biostatistical support for this study; to Ashley Kairalla and Douglas Parker for research coordination; to Lisa Brown for assistance with manuscript preparation; and to Pari Respiratory Equipment for supplying nebulizers and compressors used in this study.
REFEREN CES

1. Tarran R, Grubb BR, Gatzy JT, Davis $\mathrm{CW}$, Boucher RC. The relative roles of passive surface forces and active ion transport in the modulation of airway surface liquid volume and composition. J Gen Physiol 2001;118:223-36.

2. Matsui H, Grubb B, Tarran R, et al. Evidence for periciliary liquid layer depletion, not abnormal ion composition, in the pathogenesis of cystic fibrosis airways disease. Cell 1998;95:1005-15.

3. Mall M, Grubb BR, Harkema JR, O'Neal WK, Boucher RC. Increased airway epithelial $\mathrm{Na}+$ absorption produces cystic fibrosis-like lung disease in mice. Nat Med 2004;10:487-93.

4. Robinson M, Regnis JA, Bailey DL, King M, Bautovich GJ, Bye PT. Effect of hypertonic saline, amiloride, and cough on mucociliary clearance in patients with cystic fibrosis. Am J Respir Crit Care Med 1996;153:1503-9.

5. Robinson M, Hemming AL, Regnis JA, et al. Effect of increasing doses of hypertonic saline on mucociliary clearance in patients with cystic fibrosis. Thorax 1997; 52:900-3. 
6. Eng PA, Morton J, Douglass JA, Riedler J, Wilson J, Robertson CF. Short-term efficacy of ultrasonically nebulized hyper tonic saline in cystic fibrosis. Pediatr Pulmonol 1996;21:77-83.

7. Ballmann $\mathrm{M}$, von der Hardt H. Hy pertonic saline and recombinant human DNase: a randomised cross-over pilot study in patients with cystic fibrosis J Cyst Fibros 2002;1:35-7.

8. Tarran R, Grubb BR, Parsons D, et al. The CF salt controversy: in vivo observations and therapeutic approaches. Mol Cell 2001;8:149-58.

9. Quittner AL, Sweeny S, Watrous M, et al. Translation and linguistic validation of a disease-specific quality of life measure for cystic fibrosis. J Pediatr Psycho 2000;25:403-14.

10. Sood N, Bennett WD, Zeman K, et al. Increasing concentration of inhaled saline with or without amiloride: effect on mucociliary clearance in normal subjects. Am J Respir Crit Care Med 2003;167:158-63.

11. Alexis NE, Hu SC, Zeman K, Alter T,
Bennett WD. Induced sputum derives from the central airways: confirmation using a radiolabeled aerosol bolus deliver technique. Am J Respir Crit Care Med 2001;164:1964-70.

12. Mortensen J, Hansen A, Falk M, Nielsen IK, Groth S. Reduced effect of inhaled beta 2-adrenergic agonists on lung mucociliary clearance in patients with cystic fibrosis. Chest 1993;103:805-11.

13. American Thoracic Society. Standardization of spirometry, 1994 update. Am J Respir Crit Care Med 1995;152:1107 36.

14. Ma T, Thiagarajah JR, Yang $\mathrm{H}$, et al Thiazolidinone CFTR inhibitor identified by high-throughput screening blocks cholera toxin-induced intestinal fluid secre tion. J Clin Invest 2002;110:1651-8.

15. Narvarte J, Finn AL. Anion-sensitive sodium conductance in the apical mem brane of toad urinary bladder. J Gen Physiol 1980;76:69-81.

16. Matsui H, Davis CW, Tarran R, Boucher RC. Osmotic water permeabilities of cultured, well-differentiated normal and cystic fibrosis airway epithelia. J Clin Invest 2000;105:1419-27.

17. Wills PJ, Hall RL, Chan W, Cole PJ. Sodium chloride increases the ciliary transportability of cystic fibrosis and bronchiectasis sputum on the mucus-depleted bovine trachea. J Clin Invest 1997;99:913.

18. Verdugo P. Mucin exocytosis. Am Rev Respir Dis 1991;144:S33-S37.

19. Elkins MR, Robinson M, Rose BR, et al. A controlled trial of long-term inhaled hypertonic saline in patients with cystic fibrosis. N Engl J Med 2006;354:229-40.

20. Tarran R, Button B, Picher M, et al. Normal and cystic fibrosis airway surface liquid homeostasis: the effects of phasic shear stress and viral infections. J Biol Chem 2005;280:35751-9.

21. Camner P, Jarstrand C, Philipson K. Tracheobronchial clearance in patients with influenza. Am Rev Respir Dis 1973; 108:131-5.

Copyright (c) 2006 Massachusetts Medical Society. of Medical Journal Editors plan to consider clinical trials for publication only if they have been registered (see N Engl J Med 2004;351:1250-1).

The National Library of Medicine's www.clinicaltrials.gov is a free registry, open to all investigators, that meets the committee's requirements. 